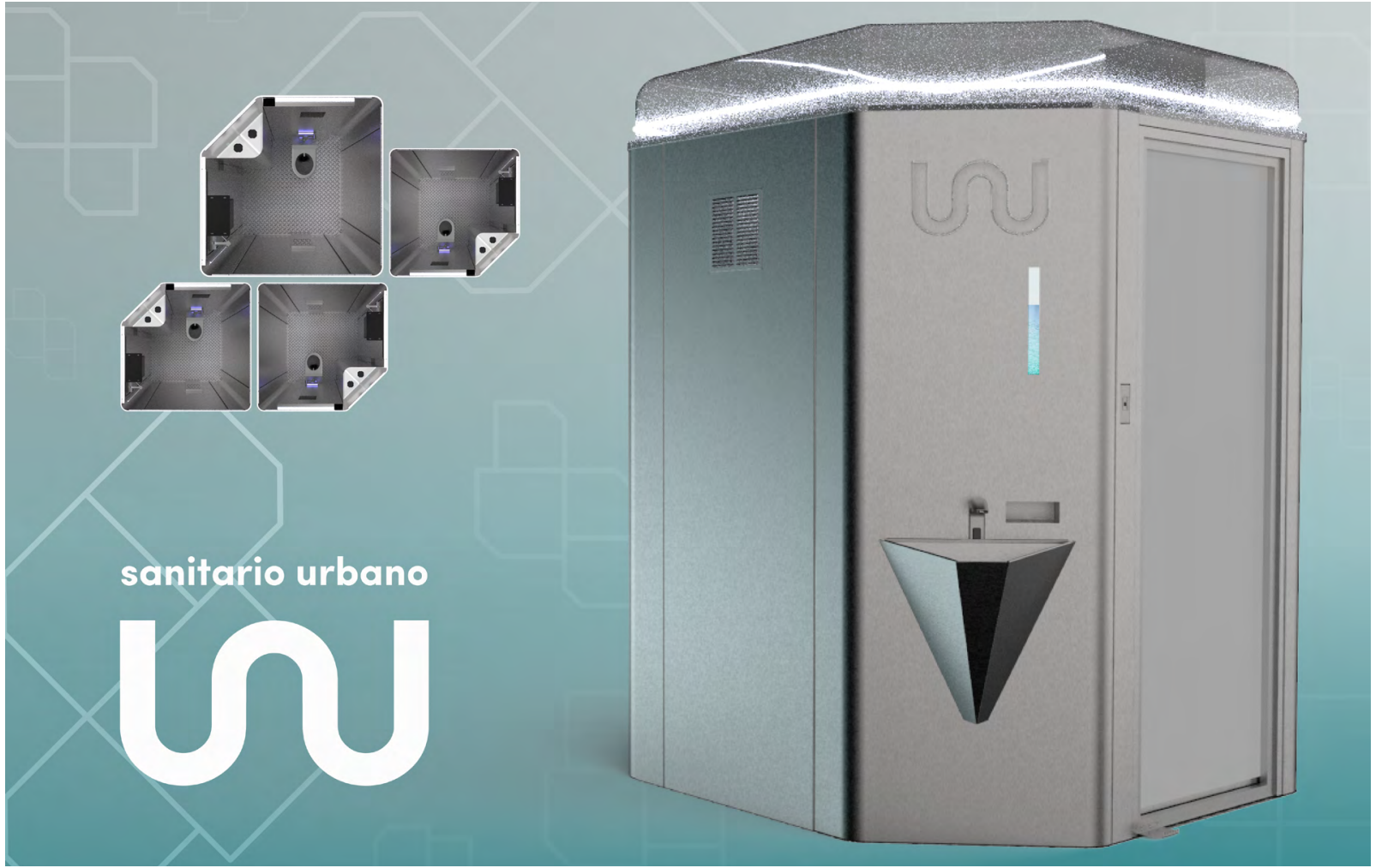

\title{
Diseño de sanitario urbano inteligente para suplir necesidades fisiológicas de personas en condición de calle en Costa Rica
}

Smart urban toilet design to meet physiological needs for homeless people in Costa Rica

Andrea M. Rojas-Jiménez ${ }^{1}$, Katherina Jurburg-Chaves ${ }^{2}$

A. Rojas-Jiménez, K. Jurburg-Chaves, “Diseño de sanitario urbano inteligente para suplir necesidades fisiológicas de personas en condición de calle en Costa Rica", IDI+, vol. 4, no 1, Jul., pp. 4-21, 2021.

doi) https://doi.org/10.18845/ridip.v4i1.5773

Fecha de recepción: 20 de enero de 2021 Fecha de aprobación: 22 de febrero de 2021

1. Andrea M. Rojas-Jiménez Estudiante de Ingeniería en Diseño Industrial Instituto Tecnológico de Costa Rica Cartago, Costa Rica androji.13@gmail.com (D) 0000-0001-5613-6984
2. Katherina Jurburg-Chaves Estudiante de Ingeniería en Diseño Industrial Instituto Tecnológico de Costa Rica Cartago, Costa Rica yjurburg@gmail.com

(iD) 0000-0003-3213-935X 


\section{Resumen}

En la capital de Costa Rica, se concentra el mayor porcentaje de personas en condición de calle. Este sector de la población es marginado por la sociedad y presenta deficiencias para suplir sus necesidades básicas. Por lo cual, durante el proyecto, se estudian estas carencias para determinar cuál puede solucionarse a través del diseño de un producto inteligente que mejore su calidad de vida.Debido a lo anterior, se realiza una etnografía digital para obtener información sobre el usuario final y se investigan sus necesidades de mayor importancia. Una vez identificadas dichas necesidades, se decide desarrollar un producto para el manejo de los desechos sanitarios de esta población; este cubre necesidades biológicas, de seguridad y de pertenencia. Para el desarrollo del producto, se lleva a cabo una conceptualización que contempla la definición y validación de requisitos, según la necesidad planteada, un estudio de mercado y un análisis de lo existente.

Una vez planteados estos aspectos, se definen sus funciones, tanto prácticas como inteligentes, componentes, perceptualidad, ergonomía, topología, interacción, ensamble, instalación, materiales y manufactura. Esto da como resultado a SU, un sanitario urbano inteligente desarrollado mediante modelado 3D y renderizado de alta calidad. En este diseño, se limita el contacto con el usuario y se plantea una desinfección automática periódica con ayuda de sensores y luz. Además, este es un producto modular de acero inoxidable, lo que facilita su fabricación y montaje de varios sanitarios. Este producto aporta social e individualmente, ya que su implementación reduce de manera indirecta los desechos sanitarios en las zonas públicas y beneficia directamente a los habitantes de la calle.

\section{Palabras Clave}

Servicio sanitario público; indigencia; baño inteligente; producto inteligente; automático

\section{Abstract}

The city of San José has the highest rate of homeless people in Costa Rica. This sector of the population is usually marginalized by society and has deficiencies when it comes to meeting their basic needs. During the project, these deficiencies were studied in order to define which ones could be solved through the design of a smart product, with the idea of improving their quality of life.A digital ethnography was carried out with the purpose of obtaining relevant information on the subject. The most important needs were investigated, based on that, the decision to develop a sanitary waste management product was made, which involves biological, security and belonging needs. 
The development of the product required a design concept that included the definition and validation of requirements according to the need specified, a market study and the analysis of existing analog products.

Once those aspects were defined, the practical and smart functions were detailed along with the needed components, perception, ergonomics, shape, interaction, assembly, installation, materials and their respective manufacturing processes. This resulted in SU, a smart urban toilet developed by using high-quality 3D modeling and rendering. This design limits the contact product-user and raises a periodical automatic disinfection by using sensors and light. Besides, it's a modular stainless steel product, which makes it fabrication and multiple assembly easier. This product represents a social and individual contribution of design, it's implementation indirectly reduces the pollution for sanitary waste in public areas and benefits homeless people directly.

\section{Keywords}

Public toilet; homeless; smart restroom; smart product; automatic

\section{Introducción}

En Costa Rica habitan alrededor de 3753 personas en situación de calle, según datos del Sistema de Información de Población Objetivo del Instituto Mixto de Ayuda Social (IMAS) [1]. Como muestra el gráfico A, el 90\% de estas personas son hombres [2], pues los varones se refugian en sustancias adictivas con más frecuencia y no buscan apoyo emocional [3].

La mayoría de estas personas se concentra en la capital del país, en la Zona Noroeste del casco central conocida popularmente como Zona Roja; en el gráfico B, se puede observar la distribución por provincia [3]. En esta zona hay ciertas particularidades del interés de dicha población que les facilitan su supervivencia. Es un lugar muy transitado, por lo que pueden pedir ayuda a los transeúntes. Así mismo, la presencia de cuatro mercados, cantinas y bares les permite conseguir alcohol y comida. Además, los lotes baldíos y casas abandonadas son un buen lugar para dormir.

Como se representa en el gráfico $\mathrm{C}$, la mayoría de las personas en condición de calle tiene educación formal [4], pese a lo que se suele creer por los prejuicios sociales. Además, en [5], el $90 \%$ de personas empadronadas en el instituto tienen de 18 a 64 años, por lo que se encuentran en edad reproductiva como lo muestra el gráfico D.

Actualmente, el centro municipal de San José ofrece el dormitorio municipal, aseo, alimentación, atención médica y otros servicios para esta población. Lastimosamente (véase gráfico $\mathrm{E}$ ), por cada 30 habitantes de la calle que se ubican en San José, solo una persona puede recibir atención en este centro [6]. 

A. Sexo [2]
C. Escolaridad [4]

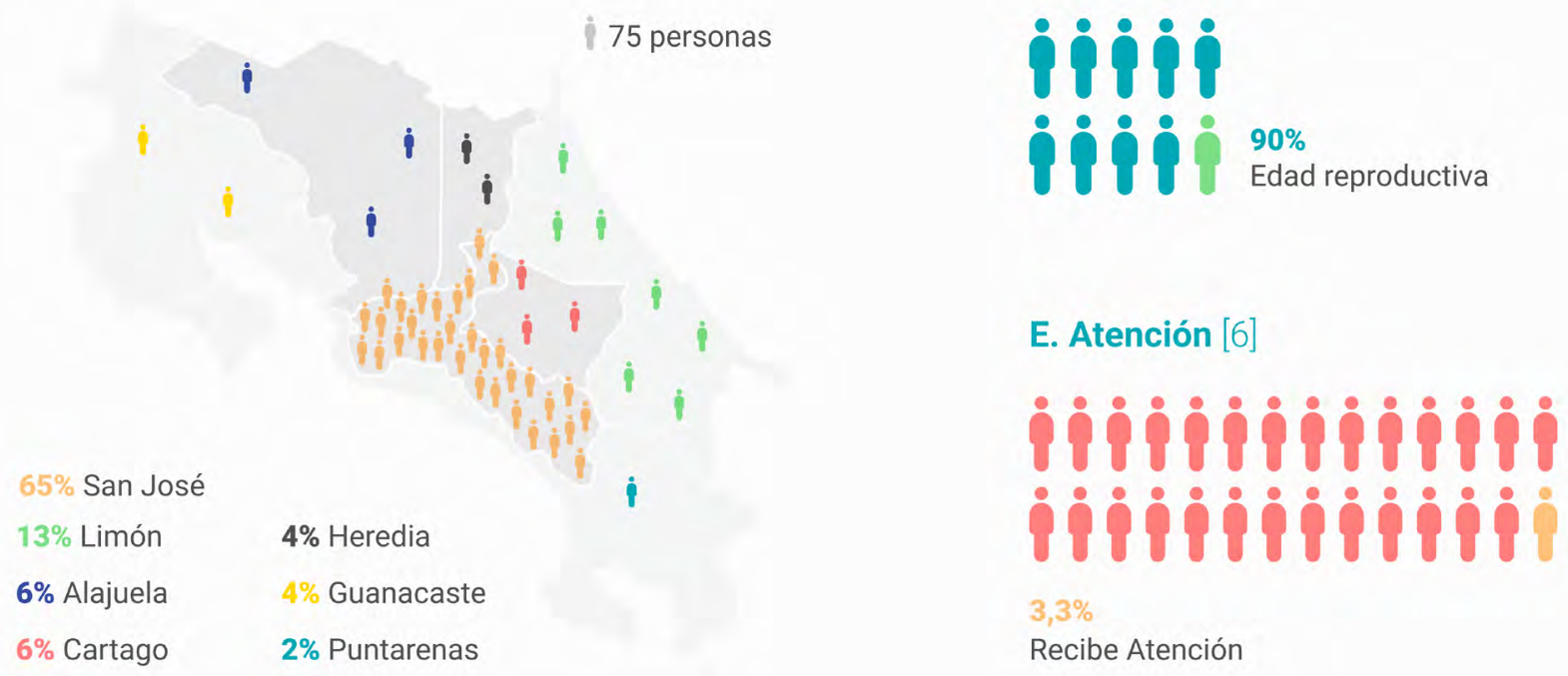

Figura 1. Información cuantitativa de la indigencia en Costa Rica Fuente: Elaboración propia, 2020.

Los principales problemas identificados en el desarrollo del proyecto se relacionan con el acceso deficiente que tienen las personas en condición de calle para suplir sus necesidades básicas, entre las cuales se encuentran las de tipo fisiológico, de seguridad y de pertenencia. Ignorar estas necesidades resulta en fatalidad, depresión y marginación.

Para este proyecto, se decide reducir el enfoque a las siguientes necesidades:

\section{Fisiológicas}

Se plantea sustentar estas necesidades haciendo énfasis en la higiene y la necesidad de excreción, por ende, se decide desarrollar el diseño de un servicio sanitario.

\section{De seguridad}

Las personas en condición de calle se exponen a distintos factores que atentan contra su seguridad, al realizar sus necesidades fisiológicas a la intemperie, debido a que buscan lugares 
alejados y solitarios para este fin. Por ende, se requiere un espacio para que puedan hacerlo de forma segura

\section{De pertenencia}

El no poseer un espacio privado para realizar sus necesidades fisiológicas genera un sentimiento de rechazo en la población hacia los habitantes de la calle. Esto fomenta la marginalización de este sector.

Tras considerar los puntos mencionados anteriormente, junto con los antecedentes y datos estadísticos de las personas en situación de calle, se define el objetivo de la investigación: resolver las necesidades fisiológicas, de seguridad y pertenencia de esta población en Costa Rica de forma inteligente, salubre, segura y privada, a través del desarrollo de un producto inteligente para entornos urbanos.

El desarrollo de este proyecto da como resultado un aporte social, pues plantea mejorar la salubridad de las zonas de la Gran Área Metropolitana (GAM) donde se concentra la población en estudio. Por otro lado, se da un aporte individual, ya que beneficia a los individuos que conforman esta población, al suplir una de sus necesidades básicas de forma digna. Además, es un aporte al diseño inclusivo, pues se diseña de forma empática, pensando en quienes viven en situación de pobreza y han sido excluidos, tachados como "pobres", "vagabundos", "mendigos", "vagos", “ladrones" o "criminales"[7].

\section{Materiales y métodos}

\section{Exploración y definición de la problemática}

Al iniciar con el proyecto se propone diseñar un producto bajo el concepto de una ciudad inteligente. Para delimitar el tema por desarrollar, se llevó a cabo una lluvia de ideas con problemáticas actuales en entornos urbanos, que fueron posteriormente clasificadas y evaluadas del 1 al 5 ante distintos criterios en una matriz, las cuales son, en orden de importancia: su alcance, posibilidad de incorporar Tecnologías de la Información y la Comunicación (TICS), el acceso que se tenía para recolectar información de este tema (dado el tiempo disponible y contexto en el que se desarrolló el proyecto, la pandemia por COVID-19) y la posibilidad de innovación.

Como resultado de dicha matriz, se exploraron las problemáticas que rodean a las personas en situación de calle, puesto que fueron las que obtuvieron un mayor porcentaje de importancia en comparación con otras ideas. Contemplando el difícil acceso a la información, se decidió realizar una investigación digital para hallar datos sobre las necesidades de los futuros usuarios del producto. 
Distintos autores aseguran que, conforme se desarrollan los medios de comunicación, nacen nuevas formas de realizar estudios, como es el caso de la etnografía mediada por la tecnología [8], la cual implica la utilización de entornos virtuales con el fin de conseguir tanto aspectos cualitativos como cuantitativos de la investigación, en búsqueda de varios conceptos.

Se elige este método para trabajar, además, tomando en cuenta la información disponible en línea con respecto al tema, se dio una gran prioridad a la técnica de observación. A través de ella, se analizó el contenido de seis vídeos encontrados en la web sobre la indigencia en el país, en el periodo desde 2009 hasta 2020. Este estudio se sistematizó con la ayuda de una ficha de observación, en la cual se anotaron testimonios (tanto de futuros usuarios del producto como de transeúntes) y observaciones.

Tomando en cuenta la recurrencia de estos datos dentro de los videos, se concluyeron hallazgos relevantes para el proyecto, con respecto a la forma de vida de las personas en situación de calle. Estos datos se resumen en la figura 2, con ellos se definió al usuario, su entorno y sus necesidades.

Resumen etnografía digital

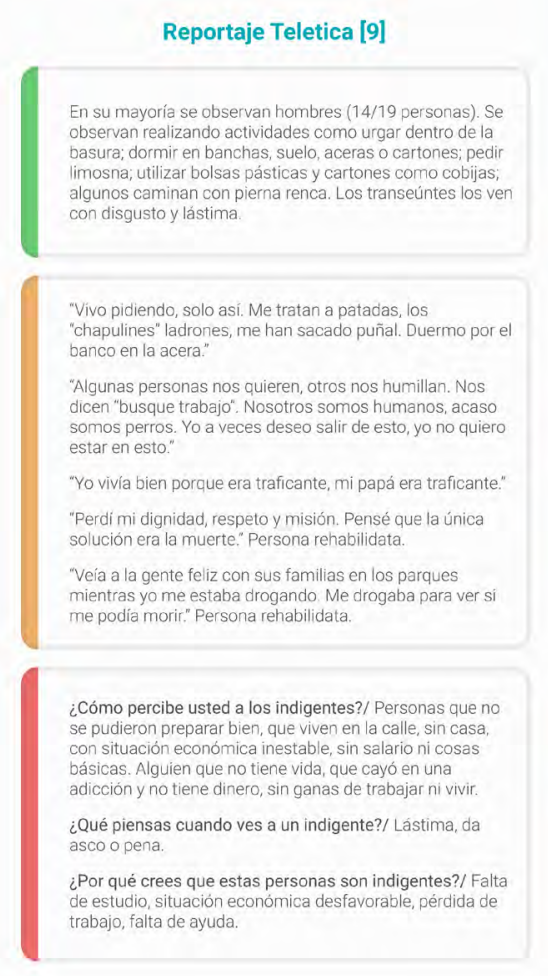

Reportaje Repretel [10]

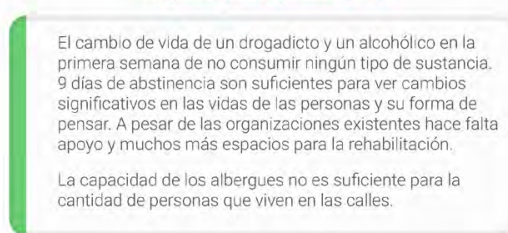

"Es importante que se sigan abriendo centros y lugares en los cuales puedan ayudar a personas que realmente lo ocupan"

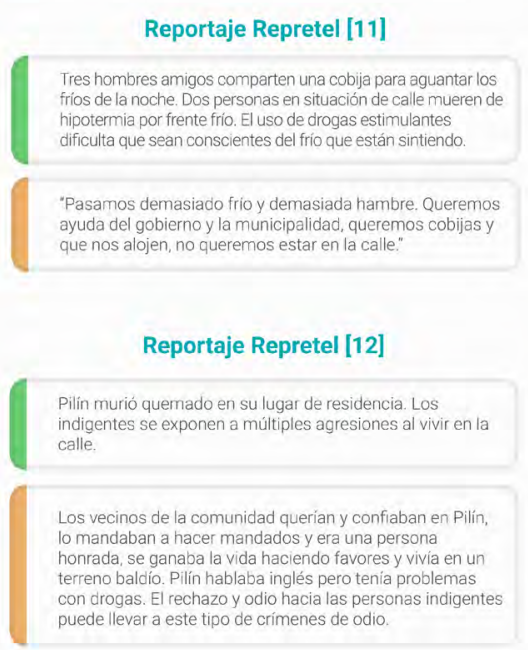

Entrevista Johnna Gónzalez [13]

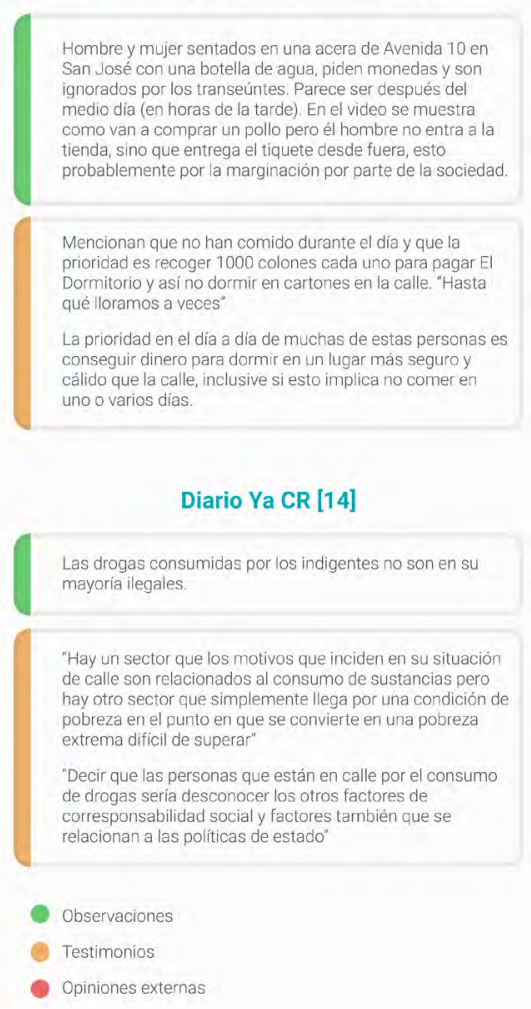

Figura 2. Resumen etnografía digital

Fuente: Elaboración propia, 2020. 
A partir de las necesidades del usuario, se procede a definir los requerimientos junto con los requisitos del producto por diseñar, mediante un árbol de problemas con el cual se organizaron y clasificaron las principales necesidades de los usuarios. Posteriormente, fueron evaluadas en una matriz y se les dio prioridad de acuerdo con la Pirámide de Necesidades de Maslow.

Posterior a esto, los requisitos y requerimientos definidos fueron validados con 34 personas a través de una encuesta digital a transeúntes del entorno de estudio. Estos resultados se contrastaron con la evaluación anterior para identificar las de mayor importancia.

Una vez identificadas, se decide trabajar la necesidad de manejo de desechos sanitarios, debido a que es la necesidad menos atendida en esta población, y no satisfacerla es negativo tanto para los usuarios como para los transeúntes.

\section{Definición de la funcionalidad}

Se definieron las funciones inteligentes y prácticas que tendrá el producto para manejar desechos sanitarios a través del diagrama $\mathrm{FAST}^{1}$ que se muestra en la figura 3 . Al tener estas funciones definidas, se investigaron componentes electrónicos y mecánicos existentes, mecanismos de interacción, materiales y sus respectivos procesos de manufactura, así como referenciales de productos análogos para tomarlos en cuenta a la hora de desarrollar el diseño externo e interno del sistema.

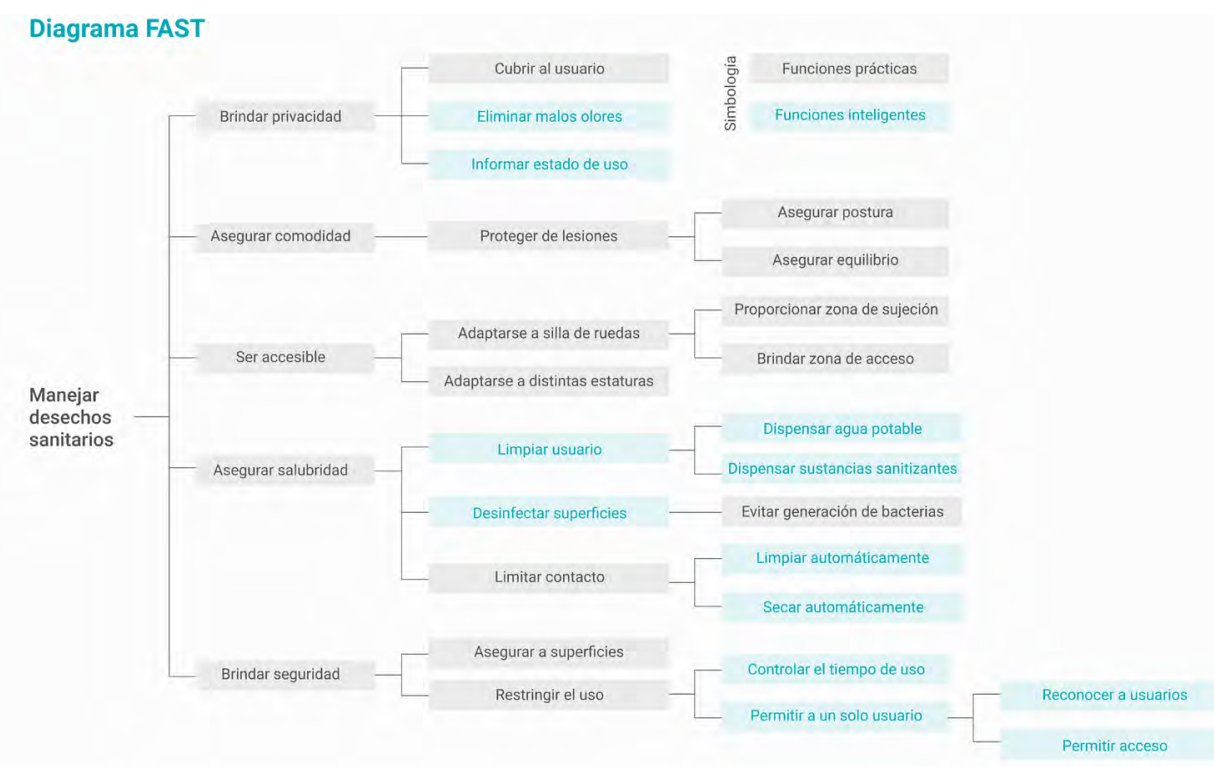

Figura 3. Diagrama FAST

Fuente: Elaboración propia, 2020.

1 Un diagrama FAST es una descripción funcional de un sistema técnico. La lectura de derecha a izquierda indica cómo las funciones de la izquierda están siendo producidas. La lectura de izquierda a derecha indica por qué las funciones a la derecha son desempeñadas [15]. 
Se analizaron los posibles componentes del sistema y, a través de una matriz, se definió cuáles eran convenientes para el proyecto. Por medio de un diagrama de flujo, se propuso cómo será el funcionamiento del producto y su relación con la entrada o salida de información, que se dará a través de la interacción del usuario con este.

Posteriormente, se definieron los sistemas y subsistemas que conformaron el producto para manejo de desechos sanitarios, tomando en cuenta los componentes elegidos previamente.

\section{Definición de la forma}

Una vez definidas las funciones inteligentes y prácticas que realizará el producto a través del diagrama FAST y el esbozo del producto utilizando el esquema de los sistemas y subsistemas, se hizo la primera aproximación formal del producto por medio de la realización de bocetos rápidos de calidad estructural, para previsualizar una posible distribución de los componentes en el sistema.

Con estos bocetos se evaluó el producto con siete personas que suelen transitar por las zonas donde se ubica el público meta. Es importante aclarar que, con cada boceto, se realizó una propuesta superficial de los materiales, considerando propiedades físicas como dureza, flexibilidad, precio y masa, así como los procesos de manufactura que hacen posible su fabricación; todos estos datos se terminan de detallar en la siguiente etapa, sin embargo, fue necesario explorar al respecto para delimitar los aspectos generales de la topología.

Como se indica en la figura 4 , se utilizó el método de objetivos ponderados ${ }^{2}$, se seleccionó la propuesta con mayores beneficios para el usuario, así como más sencilla en su fabricación y modularidad.

2 Se utiliza una matriz que clasifica las propuestas frente a criterios importantes para el éxito del producto, a estos se les asigna un número según su importancia. El equipo de diseño califica al producto según los criterios seleccionados en una escala de 1 al número de importancia de cada criterio. Posteriormente, se suma el puntaje de cada propuesta y la que posea el mayor puntaje es la propuesta seleccionada [16]. 


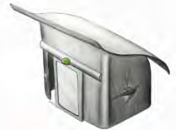

Propuesta 1

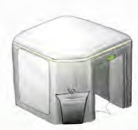

Propuesta 2

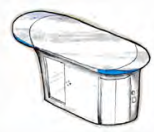

Propuesta 3

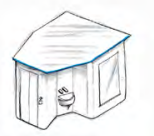

Propuesta 4
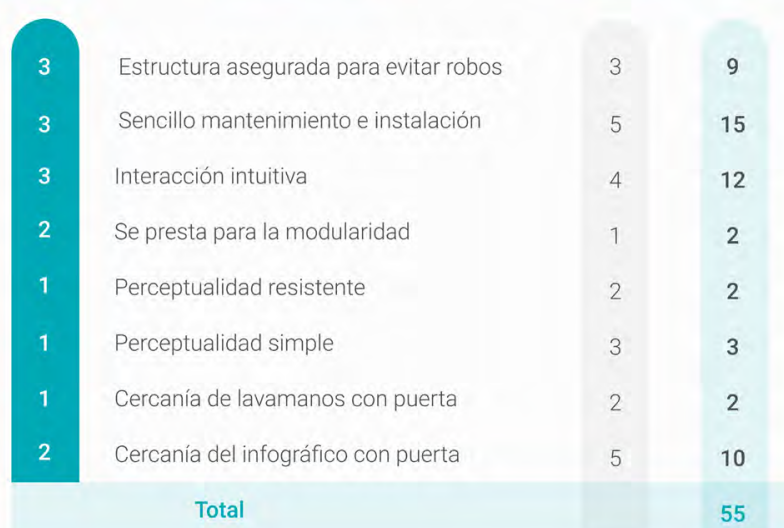

Figura 4. Selección de la propuesta

Fuente: Elaboración propia, 2020.

Una vez seleccionada la propuesta con la que se continuaría trabajando, se hizo un modelado 3D volumétrico con el fin de evaluar la interacción con el usuario y la configuración de los componentes del producto. Se tomaron en cuenta nuevos factores relevantes para el diseño, evaluados con este modelo de baja fidelidad. Se procedió a detallar el modelo, además, se consideró el proceso de manufactura seleccionado (plegado de lámina metálica) para el modelado de las paredes externas, a las cuales en conjunto se les llamará carcasa durante el desarrollo del artículo, para facilitar la comprensión de la estructura del producto.

\section{Definición de la manufactura}

A partir del modelo 3D final, se concretaron los materiales por utilizar para las piezas y, posteriormente, se hicieron los planos de cada panel que compone la carcasa metálica. Así mismo, se realizaron los planos de las otras piezas de fabricación propia como lo son el techo, el fregadero y los tanques para fluidos.

Durante esta etapa, se reunieron, en una lista con sus respectivos precios y fichas técnicas, todos los componentes prefabricados. Además, se realizó un mapeo del proceso de manufactura e instalación, que se basa en una combinación de ensamblaje dentro y fuera de la planta de fabricación para facilitar el transporte y la instalación del producto. 


\section{Simulación del diseño}

Debido a las implicaciones de la pandemia causada por el COVID-19, la modalidad virtual de los cursos de diseño y, por ende, la imposibilidad de realizar prototipos físicos y validaciones con usuarios reales, se concluyó la investigación realizando simulaciones del producto en el software Keyshot, tanto en forma de imágenes de alta calidad como en animaciones que emulan el ensamblaje del producto, la interacción con el usuario y algunas funciones como las luces de estado, iluminación general y desinfección UV.

Estas simulaciones se compilaron en un vídeo informativo que explica con detalle el diseño del producto, desde el concepto de diseño, hasta detalles técnicos como la configuración de los componentes, el funcionamiento y la interacción con el usuario.

\section{Discusión de resultados}

A partir de la investigación etnográfica digital realizada en la primera etapa, se obtuvo información sobre los usuarios, sus necesidades, entorno y problemáticas, pues en los videos analizados varios involucrados se expresaron al respecto, dos de ellos dieron su testimonio tras haber salido de la situación de indigencia. Las personas en condición de calle pasan calamidades, son marginadas por la sociedad y carecen de necesidades básicas en general. Debido a la vida precaria de esta población, se eligieron como usuarios finales.

Se identificaron numerosas necesidades en esta población, tales como refugio, apoyo, comida, cobijo, empatía, sustento, seguridad, higiene, rehabilitación, salud y trabajo. No obstante, se consideraron como primordiales las que involucran el acceso a suplir sus necesidades básicas.

Existen iniciativas como Chepe se baña, que proporcionan un espacio donde estas personas pueden asearse. Con este programa y la ayuda de voluntarios, atienden 100 personas por día [17], sin embargo, este se centra en el baño y no así en los servicios sanitarios. Por lo que se optó por desarrollar un producto para manejar los desechos sanitarios y que se encuentre siempre a su disposición.

El entorno definido para el proyecto es, principalmente, el cantón central de San José, pues allí se encuentra el $65 \%$ de estas personas [3], aunque puede extenderse a las cabeceras y cantones centrales del país, donde, por lo general, se concentra esta población. La cantidad de SU en cada zona estaría sujeta a la densidad de usuarios en ellas.

En la etapa inicial, se deseaba resolver muchas problemáticas con un único producto, lo cual resultaba complejo y poco realista tomando en cuenta el tiempo disponible para desarrollar el proyecto. La segunda etapa permitió delimitar las funciones del producto para así conseguir una solución más focalizada y, por lo tanto, con mayor nivel de detalle para el problema planteado. 
En la tercera etapa, se hizo la primera evaluación del producto con siete personas externas al proyecto, quienes suelen transitar por el entorno definido. Esto permitió obtener información de utilidad que no se había considerado hasta el momento en el diseño, de modo que el flujo propuesto para el uso del producto se fue modificando para atender a estos nuevos hallazgos.

A la hora de definir la manufactura, se realizó una amplia investigación con respecto al plegado de lámina metálica. Esta etapa del proceso se convirtió en el punto de mayor aprendizaje durante el diseño del producto, debido a que se manejó por primera vez con profundidad, por parte de las diseñadoras, un producto basado en la manufactura.

El mayor reto en esta etapa fue trabajar con los tamaños de láminas metálicas que se encuentran en el mercado, ya que estas tienen dimensiones menores a las paredes del cubículo. Se decide juntar varias láminas plegadas para completar el perímetro de la carcasa, esto permitió proteger al usuario del filo del material y actuar como soporte estructural. La unión se dio soldando pestañas en forma de "C" que fueron plegadas en las láminas como se observa en la figura 5.

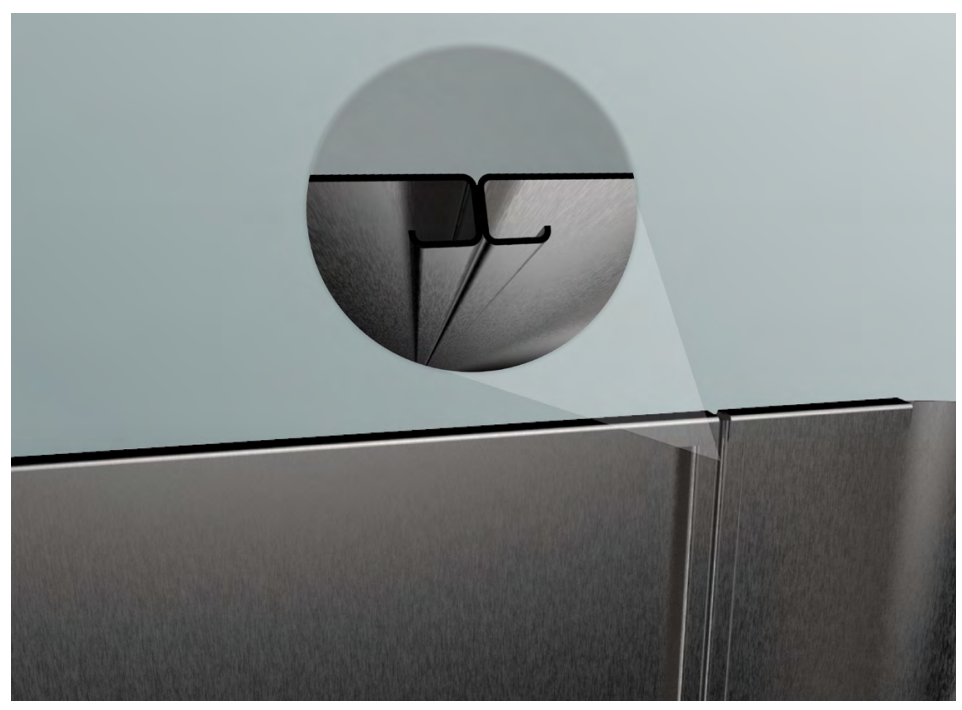

Figura 5. Unión estructural entre láminas

Fuente: Elaboración propia, 2020.

La iluminación interna y externa de los cubículos significó un reto para el diseño de SU, debido a que se deseaba optimizar la luz en estos. Se decidió cambiar el techo de acero original por uno de policarbonato, esto, además de permitir el paso de la luz natural durante el día hacia adentro de los baños, permitió que la manguera LED utilizada como luz interna funcionara, a su vez, para iluminar el exterior por las noches.

Esta iluminación nocturna brinda seguridad a los usuarios y a los peatones. Asimismo, otro valor agregado del techo traslúcido es la reducción en el peso de este y la percepción de un espacio más ameno [18] al usuario, debido a la luz natural. En la figura 6, se observa de forma gráfica la solución a este problema. 


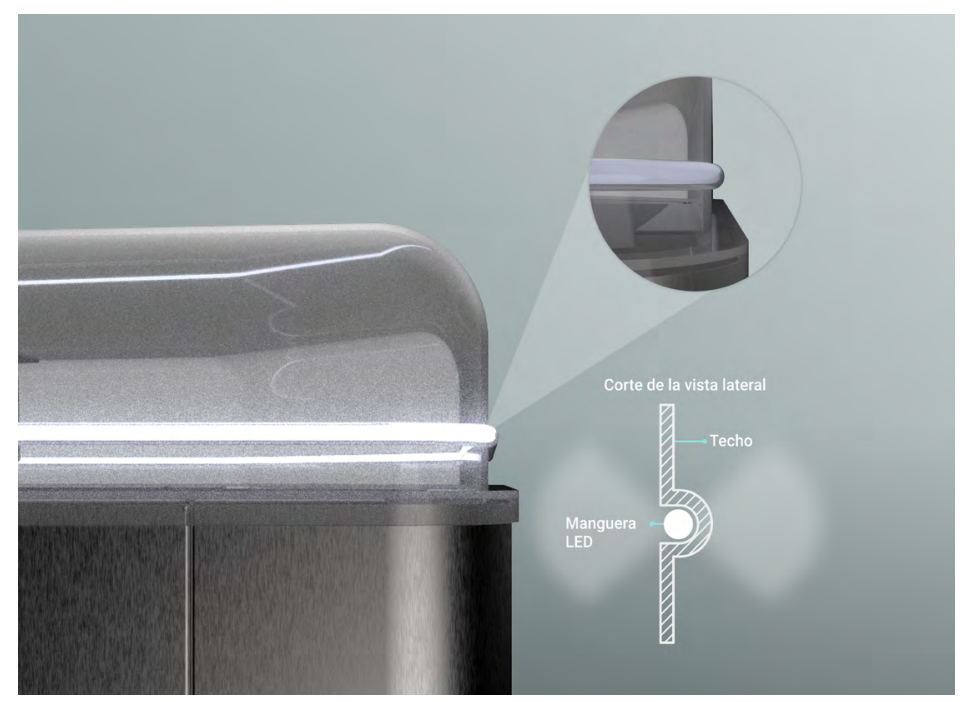

Figura 6. lluminación de los cubículos

Fuente: Elaboración propia, 2020.

La unión entre el techo y la carcasa representó otro obstáculo, debido a que el espesor de 19 $\mathrm{mm}$ de las láminas de acero no podría resistir el peso y tamaño del techo, si este se colocaba canto con canto. Este factor se solucionó agregando una pestaña de $2,5 \mathrm{~cm}$ que se extiende perpendicular a las láminas hacia el interior del cubículo; sobre esta se coloca el techo, el cual tiene también esta pestaña en el sentido contrario seguida por una perpendicular hacia abajo, en la que se colocan los tornillos respectivos. La solución de esta unión se explica de forma gráfica en la figura 7.

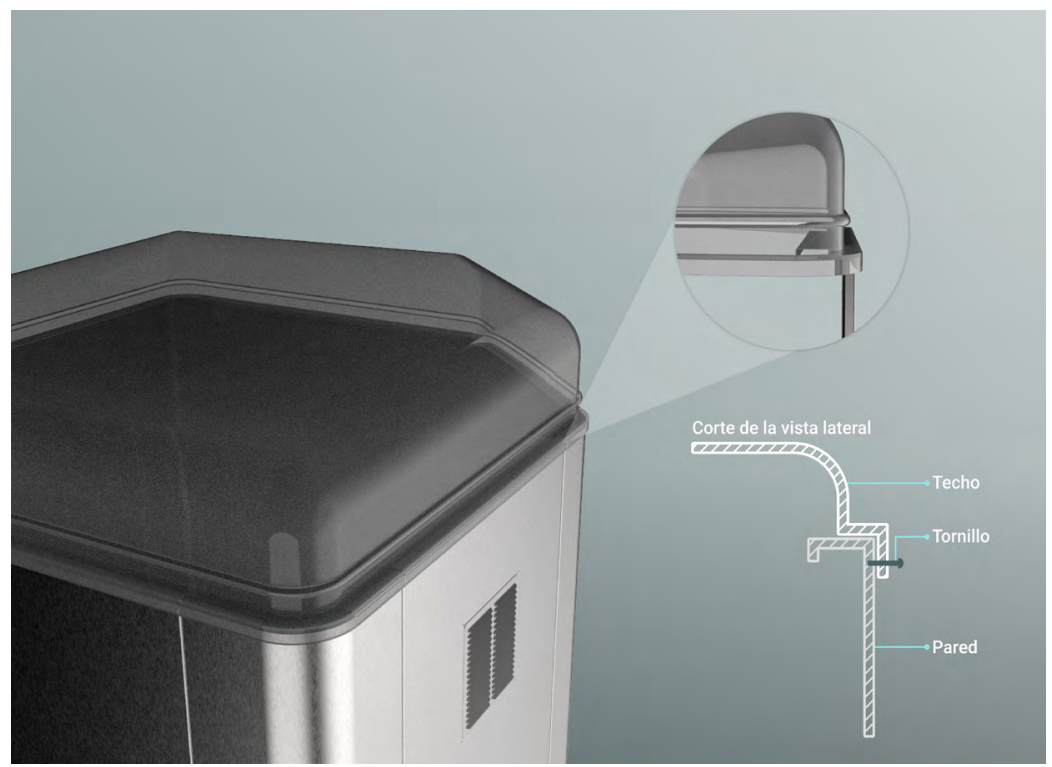

Figura 7. Unión estructural entre la carcasa y el techo Fuente: Elaboración propia, 2020. 
Los obstáculos resueltos fueron de trascendencia, no solo para el proceso de diseño, sino que se convirtieron en una experiencia de aprendizaje, a partir de la cual se abren puertas para brindar soluciones a problemas similares en el futuro e incluso mejorar las soluciones propuestas para este proyecto.

En la figura 8, se detallan las funciones tanto inteligentes como prácticas que realiza el producto en el exterior. Entre las funciones inteligentes, se encuentra el acceso restringido a los usuarios, el cual se consigue utilizando un sensor biométrico, este permite que solo los usuarios con su huella dactilar registrada en una base de datos tengan acceso al interior del cubículo, a su vez, el sensor indica a través de un código de color el estado de uso del sanitario. Otra función inteligente que desempeña el producto en su exterior es la de dispensar jabón de forma automática a través de un sensor de proximidad, este mismo sistema se utiliza para abrir el tubo de agua del lavamanos.

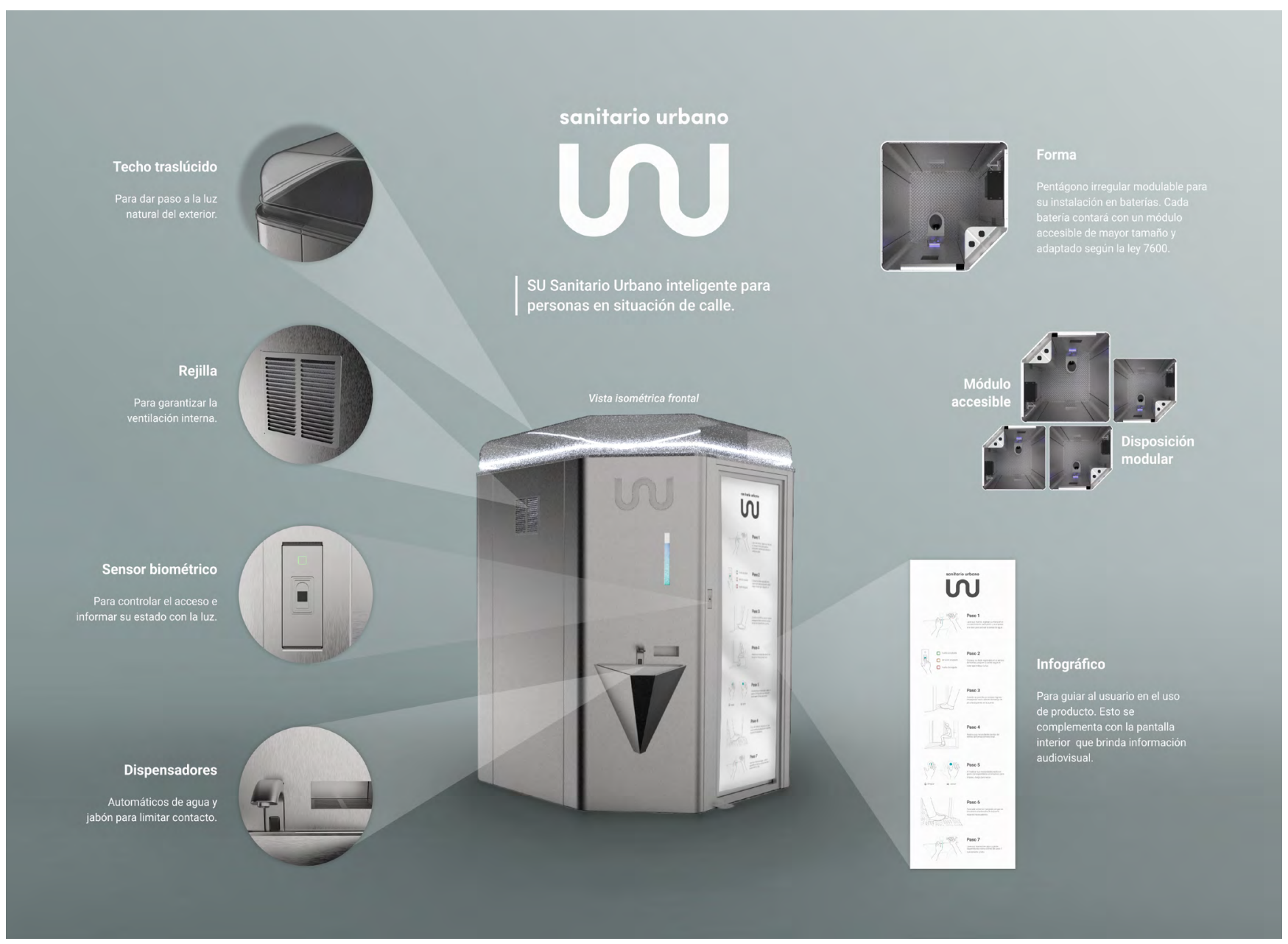

Figura 8. Infográfico de funcionamiento externo

Fuente: Elaboración propia, 2020. 
Para desempeñar las funciones prácticas, el sanitario cuenta con un techo translúcido que, como se ha mencionado anteriormente, permite el paso de la luz natural para iluminar al cubículo durante el día. También posee una rejilla que permite la circulación del aire dentro del sanitario, esto ayuda a disminuir los malos olores de una forma eficiente. En la puerta de $\mathrm{SU}$, se encuentra un infográfico con instrucciones para guiar al usuario en el uso del producto, en cuanto los usuarios consigan el uso del sanitario sin errores, este infográfico se sustituirá con un MUPI que ayudará a subvencionar el mantenimiento de las unidades en el futuro.Los sanitarios cuentan con una topología modular que permite la instalación de las unidades en baterías sanitarias, además, se planteó un módulo accesible de mayor tamaño que se adapta a la Ley 7600.

En la figura 9, se detalla la distribución interna del sanitario y las funciones finales realizadas por el producto en el interior. En la parte superior de la puerta, se encuentra un contador de personas para llevar un registro de ingresos y permitir solo una persona a la vez. A la izquierda de la entrada, se encuentra el compartimento de mantenimiento para posibles averías y suplir los tanques de fluidos de aromatizante y jabón.

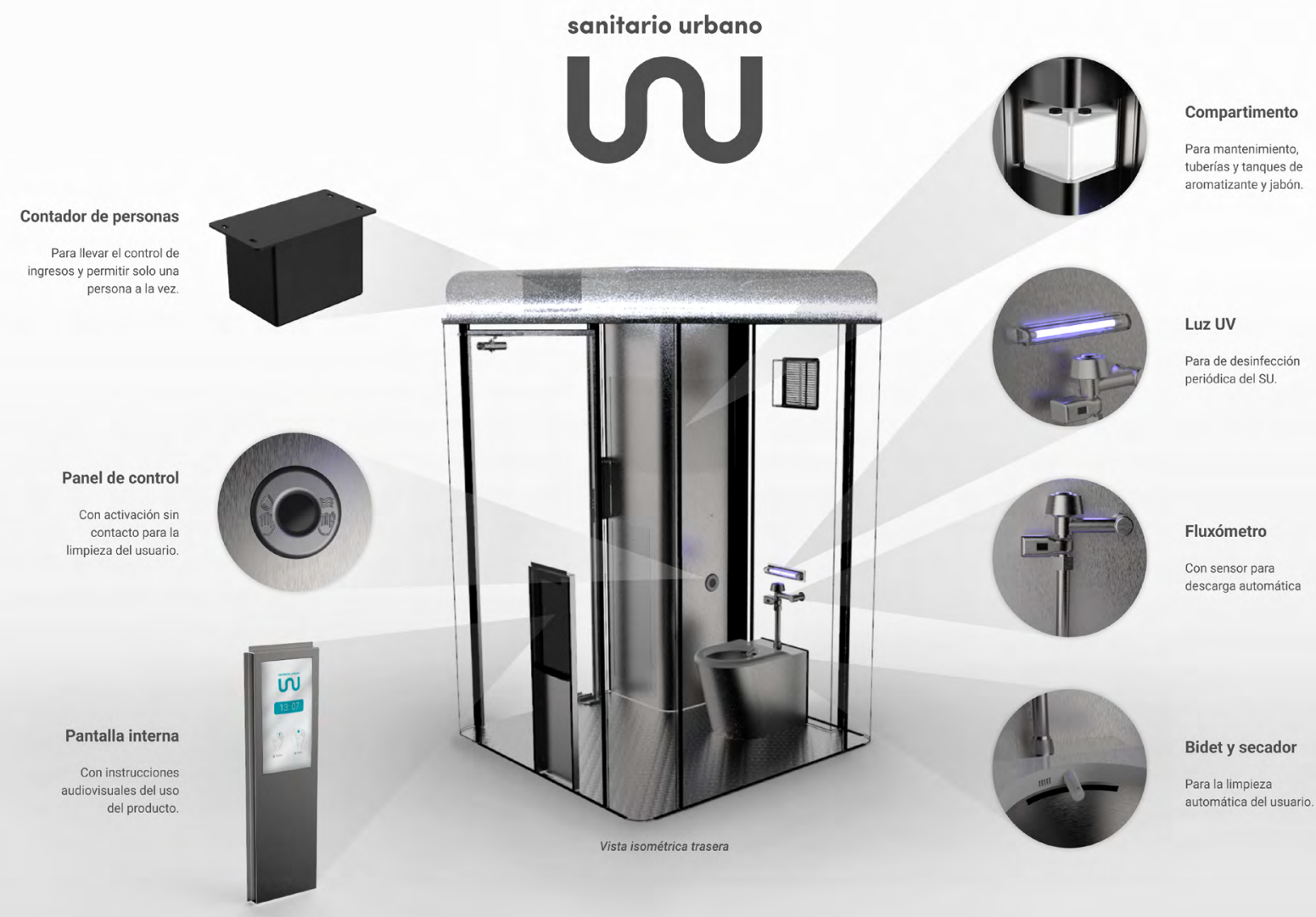

Figura 9. Infográfico de funcionamiento interno

Fuente: Elaboración propia, 2020. 
Frente al retrete se encuentra una pantalla informativa con instrucciones para su uso. Además, a la derecha del retrete, se encuentra un sensor para activar la limpieza y secado sin contacto. El retrete se compone de un fluxómetro automático con sensor, un bidé y un secador; en la parte superior se ubica una luz UV para la desinfección del servicio después de cada uso.

Durante la etapa de simulación de diseño, se definieron siete pasos para interactuar de manera óptima con el producto diseñado. Primero, el usuario se debe lavar las manos con el agua y jabón de los dispensadores automáticos externos, así tendrá las manos limpias para colocar el dedo registrado en el sensor de huella digital de la entrada y podrá ingresar. Este sensor cuenta con tres luces: la luz verde significa huella aceptada, la naranja servicio ocupado y la roja huella denegada. Cuando la huella es aceptada, el usuario debe acceder y abrir con la manija de pie que se encuentra en la parte inferior de la puerta.

Una vez en el interior, el usuario realizará sus necesidades dentro del retrete de forma convencional. Posteriormente, deberá accionar la limpieza a través de dos gestos en el sensor a su derecha, acercar la mano para activar el bidé y agitarla para secar. Al finalizar, deberá salir con la manija de pie interna y lavarse las manos nuevamente en el exterior. En la figura 10 , se muestra este proceso de forma infográfica.

Se identifican las siguientes necesidades futuras para continuar con el desarrollo del producto:

- Se debe continuar la investigación con el fin de definir el sistema de limpieza para las superficies de las unidades sanitarias, ya que es una oportunidad para generar un diseño autosostenible en su mantenimiento.

- Es necesario realizar un estudio más detallado sobre el transporte de las piezas y considerar la posibilidad de realizar el ensamblaje en la planta de manufactura, así como transportar las baterías sanitarias completamente armadas.

- Otra oportunidad para la optimización del diseño se encuentra en una mayor investigación con respecto a materiales y componentes, ya que esto podría disminuir el peso y el costo de las unidades.

- Se debe analizar la posibilidad de adaptar este proyecto a las necesidades de otros usuarios o para su colocación en entornos distintos al planteado.

Finalmente, para la optimización del diseño del producto SU, se deben contemplar las siguientes observaciones:

- Se recomienda utilizar aluminio para diseñar una carcasa más liviana y sencilla de transportar, además, se debe considerar la posibilidad de utilizar tubos metálicos como refuerzo estructural, evitando el proceso de plegado de láminas y las uniones en forma de "C".

- Para aumentar la intensidad luminosa en el interior y exterior de los módulos, colocar una manguera LED extra debajo de la existente.

- Finalmente, se debe tomar un sistema de limpieza para el suelo y paredes, el cual no fue considerado debido al corto tiempo en el que se desarrolló la investigación. 


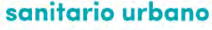

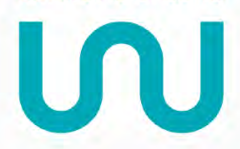 \\ Manual de usuario}

\section{Paso 1}

Lave sus manos, ingrese su mano en

el compartimento para jabón y

acerquese a la llave para activar la

salida de aqua.

\section{Paso 2}

Coloque su dedo registrado en el sensor de huellas y espere la señ según el color que indique la luz.

\section{Paso 3}

Cuando se permita su acceso ingrese empujando hacia adentro la manija de pie a la izquierda de la puerta.

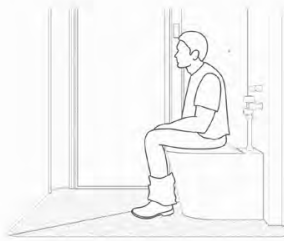

Paso 4

Realice sus necesidades dentro de retrete de forma convencional.

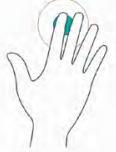

O limpiar
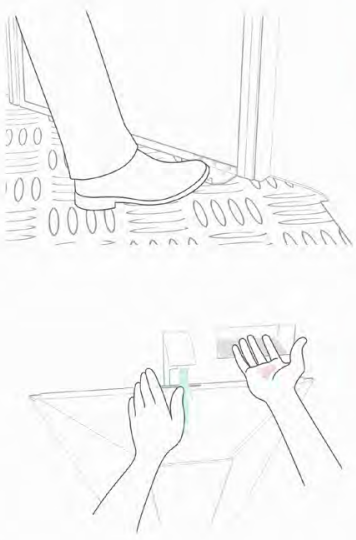

\section{Paso 6}

Para salir utilice la manija de pie que se encuentra a la derecha de la puerta halando hacia adentro

\section{Paso 7}

Lave sus manos con agua y jabón siguiendo las instrucciones del paso 1 nuevamente y listo.

Figura 10. Infográfico del manual de uso del producto

Fuente: Elaboración propia, 2020.

\section{Conclusiones y recomendaciones}

Como indica Burchardt, las sociedades latinoamericanas se caracterizan por tener las mayores tasas de desigualdad del mundo [19]. Por su parte, la formación profesional del diseñador industrial tiene el reto de responder a los desafíos de los problemas humanos [20], entre dichos problemas se encuentran la desigualdad y la pobreza.

El diseñador industrial está capacitado para reconocer las necesidades de los distintos sectores de la población y así buscar soluciones que mejoren la calidad de vida de las personas, incluso puede aportar en la reducción de las brechas sociales, al atender o resolver necesidades de quienes viven en situación de pobreza. 
Al investigar para el desarrollo de SU, se reconoció el impacto social que tienen las iniciativas que se enfocan en los usuarios en condición de calle. Esta clase de productos y servicios no solo brindan comodidad al usuario, sino que elevan y dignifican su calidad de vida, lo que resuelve más allá de sus necesidades fisiológicas y representa un aporte significativo en sus necesidades sociales.

Productos como SU contribuyen a la disminución de las brechas sociales en el mundo, pero mayormente en América Latina, donde existen menos iniciativas para reducir la tasa de desigualdad. Por lo cual, diseños como este construyen el puente que permite avanzar hacia la automatización de productos y servicios centrados en el usuario, con un enfoque hacia la equidad social, lo cual es de alta trascendencia para construir ciudades inteligentes éticas y justas.

\section{Referencias}

[1] O. Ugarte, "COVID-19 agrava la difícil vida de las personas en condición de calle", en Semanario Universidad, Mar. 2020. [En línea]. Disponible: https://semanariouniversidad. com/pais/covid-19-agrava-la-dificil-vida-de-las-personas-en-condicion-decalle/\#: :text=Al\%20igual\%20que\%20\%E2\%80\%9CEl\%20negro,Alajuela\%20y\%20 Cartago\%20tienen\%206\%25.

[2] C. Ugalde, “La indigencia en Costa Rica”, De la Mano con la Calle, Feb. 2020. [En línea]. Disponible: https://www.delamanoconlacalle.com/guias/contexto-indigencia-cr. [Acceso Sep. 23, 2020].

[3] C. Rojas, "Indigencia en San José: expresión de la exclusión social y el desarraigo", Universidad de Costa Rica, San José, Costa Rica, 2006. [En línea]. Disponible: http://www. ts.ucr.ac.cr/binarios/docente/pd-000191.pdf

[4] A. Mora, "Personas en situación de calle: la problemática invisible que es la más visible de todas", en Delfino CR, Jun.2019. [En línea]. Disponible: https://delfino.cr/2019/06/ personas-en-situacion-de-calle-la-problematica-invisible-que-es-la-mas-visible-detodas

[5] J. Solano, "Indigencia golpea al país: 90\% de ellos están en edad productiva", en CRhoy. com, Abr. 2019. [En línea]. Disponible: https://www.crhoy.com/nacionales/indigenciagolpea-al-pais-90-estan-en-edad-productiva/

[6] M. Rodríguez, "San José alberga 7 de cada 10 indigentes", en CRhoy.com, Ene. 2020. [En línea]. Disponible: https://www.crhoy.com/nacionales/san-jose-alberga-a-7-de-cada-10indigentes/

[7] P. J. Cabrera. Rodríguez, "Exclusión social: contextos para un concepto", Revista de Treball Social, Abr., pp. 9-21, 2007.

[8] M. A. Mosquera-Villegas, "De la Etnografía antropológica a la Etnografía virtual. Estudio de las relaciones sociales mediadas por Internet," Fermentum. Revista Venezolana de Sociología y Antropología, 1 de Septiembre 2008. 


\section{revistaIDI+}

[9] Teletica, "Documental sobre los indigentes en Costa Rica",Oc. 31 , 2009. [Vídeo]. Disponible en: https://www.youtube.com/watch?v=CSImNgxhUAg\&t=13s. [Acceso Oct. 01, 2020].

[10] J. González, "HOMELESS / San José / Costa Rica/ ayudando personas de la calle sin hogar/", Oct. 29 , 2016. [Vídeo]. Disponible en: https://www.youtube.com/ watch? $v=z D G j B s Q X L k c$. [Acceso Oct. 01, 2020]

[11] Noticias Repretel, “Dos indigentes murieron a causa del frente frío”, Ene. $04,2018$. [Vídeo]. Disponible en: https://www.youtube.com/watch?v=yENcRH6uFIM. [Acceso Oct. 01, 2020]

[12] Noticias Repretel, "Conocemos o que vive un drogadicto y un alcohólico en la primera semana sin consumir", Ene. 28 , 2016. [Vídeo]. Disponible en: https://www.youtube.com/ watch?v=Jj6UKGOvk1o. [Acceso Oct. 01, 2020]

[13] Noticias Repretel, “Indigentes enfrentan difícil panorama de agresión”, Feb. 19, 2015. [Vídeo]. Disponible en: https://www.youtube.com/watch?v=y2Bysdb0Mf4. [Acceso Oct. 01, 2020]

[14] Diario Ya CR, "Nota sobre Indigencia en Costa Rica”, Jul. 31, 2020. [Vídeo]. Disponible en: https://www.youtube.com/watch?v=fynY3clBE6Y. [Acceso Oct. 01, 2020]

[15] L. Rocha-Chiu, "La Ingeniería de Valor en los Proyectos de Construcción”, en Compilación de Artículos de Investigación, Azcapotzalco, Ciudad de México, Red Académica Internacional UADY, UAM, WPI, TAMU, ICA, e invitados., 2010, pp. 16-31.

[16] B. Martin and B. Hanington, Universal Methods of Design, Beverly: Rockport Publishers, 2012.

[17] A. Núñez, "Iniciativa "Chepe se baña" recibe donaciones para personas en condición de calle", en La República, Jul. 2019. [En línea]. Disponible: https://www.larepublica.net/ noticia/iniciativa-chepe-se-bana-recibe-donaciones-para-personas-en-condicion-decalle

[18] J. T. Franco, "Sin luz natural no hay buena arquitectura: ¿Cómo promover diseños moldeados y nutridos por la luz?”, Plataforma Arquitectura, 9 Diciembre, 2019. [En línea]. Disponible: https://www.plataformaarquitectura.cl/cl/926238/sin-luz-natural-no-haybuena-arquitectura-como-promover-disenos-moldeados-y-nutridos-por-la-luz. [Acceso Ene. 20, 2021].

[19] H. Burchardt, “¿Por qué América Latina es tan desigual? Tentativas de explicación desde una perspectiva inusual”, Nueva Sociedad, 1 Mayo, 2012. [En línea]. Disponible: https:// nuso.org/articulo/por-que-america-latina-es-tan-desigual-tentativas-de-explicaciondesde-una-perspectiva-inusual/. [Acceso Ene. 20, 2021].

[20] A. M. Reyes-Fabela y R. Pedroza-Flores, "Retos de la formación profesional del diseñador industrial en la Cuarta Revolución Industrial (4RI)", Revista Iberoamericana para la Investigación y el Desarrollo Educativo, Ciudad de México, 2018. 